\title{
BMJ Open Referrers' point of view on the referral process to neurosurgery and opinions on neurosurgeons: a large-scale regional survey in the UK
}

\author{
Meriem Amarouche, ${ }^{1}$ Jonathan $\mathrm{J} \mathrm{Neville},{ }^{2}$ Simon Deacon, ${ }^{2}$ Nida Kalyal, ${ }^{2}$ \\ Nikita Adams, ${ }^{2}$ Beverly Cheserem, ${ }^{3}$ Daniel Curley, ${ }^{2}$ Ruth-Mary DeSouza, ${ }^{1,4}$ \\ Fehmi Hafiz, ${ }^{2}$ Tanya Jayawardena, ${ }^{2}$ Nishi Khetani, ${ }^{2}$ Diana Matthews, ${ }^{2}$ \\ Sophie Mustoe, ${ }^{2}$ Sabrina Okafor, ${ }^{2}$ Olivia Padfield, ${ }^{2}$ Ishani Rao, ${ }^{2}$ Reem Samir, ${ }^{2}$ \\ Hyder Tahir, ${ }^{2}$ Benjamin Varghese, ${ }^{2}$ Christos Michael Tolias ${ }^{1}$
}

To cite: Amarouche M, Neville JJ, Deacon S, et al. Referrers' point of view on the referral process to neurosurgery and opinions on neurosurgeons: a large-scale regional survey in the UK. BMJ Open 2017;7:e017495. doi:10.1136/ bmjopen-2017-017495

- Prepublication history for this paper is available online. To view these files, please visit the journal online (http://dx.doi. org/10.1136/bmjopen-2017017495).

Received 27 April 2017

Revised 11 October 2017 Accepted 13 October 2017

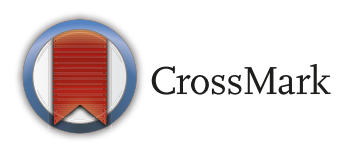

${ }^{1}$ Department of Neurosurgery, King's College Hospital NHS Foundation Trust, London, UK ${ }^{2}$ Department of Medicine, King's College London, London, UK ${ }^{3}$ Brighton and Sussex University Hospitals NHS Trust, Brighton, UK

${ }^{4}$ Department of Medicine, University College London, London, UK

Correspondence to Meriem Amarouche meriem.amarouche@gmail.com

\section{ABSTRACT}

Objectives There is an increased reliance on online referral systems (ORS) within neurosurgical departments across the UK. Opinions of neurosurgeons on ORS are extensively reported but those of referrers have hardly been sought. Our study aims at ascertaining our referring colleagues' views on our ORS and its impact on patient care, their opinions on neurosurgeons and how to improve our referral process.

Setting 14 district general hospitals and one teaching hospital.

Participants 641 healthcare professionals across a range of medical and surgical specialties including doctors of all grades, nurses and physiotherapists. Survey responses were obtained by medical students using a smartphone application.

Results Although $92 \%$ of respondents were aware of the ORS, $74 \%$ would routinely phone the on-call registrar either before or after making referrals online. The majority $(44 \%)$ believed their call to relate to a life-threatening emergency. $62 \%$ of referrers considered the ORS helpful in informing patients' care and $48 \%$ had a positive opinion of their interaction with neurosurgical registrars. On ways to improve the ORS, $50 \%$ selected email/text confirmation of response sent to referrers and $16 \%$ to referring consultants.

Conclusion Our results confirm that referrers feel that using our ORS positively impacts patient care but that it remains in need of improvement in order to better suit our colleagues' needs when it comes to managing neurosurgical patients. We feel that the promotion of neurosurgical education and mitigation of the effects of adverse workplace human factors are likely to achieve the common goal of neurosurgeons and referrers alike: a high standard in patient care.

\section{INTRODUCTION}

Referrals to tertiary services are critical in ensuring that patient care is delivered according to national standards and timeframes. Emergency departments, for
Strengths and limitations of this study

- Our study reports the opinions of referrers rather than that of neurosurgeons on the use of a regional online referral system (ORS).

- It was conducted at a regional level and only assessed opinions on a single referral system.

- It involved 15 referring hospitals and gathered the largest sample size of respondents published to date on this topic.

- Although the large number of responses obtained is likely to have captured an accurate opinion of our referrers, we cannot guarantee that these are representative of the opinions of the entire referring population. The responses were obtained using convenience sampling. Our data can therefore suffer from selection bias and, as mentioned above, may not be generalisable.

- In retrospect, the question assessing opinions on the interaction with the neurosurgery registrar as well as the one addressing the ways forward with the ORS could have been elaborated more to allow a better analysis of the results.

example, refer $20 \%-40 \%$ of their patients for a specialist opinion or admission. ${ }^{1}$ Making an accurate and safe referral can however be difficult and stressful ${ }^{2-4}$ and requires effective and efficient communication. It also requires an understanding of the factors in the history, examination and investigations that are 'rate-determining steps' in decision-making for the receiving specialty but may not be obvious points to ask for the referring doctor. Miscommunication and poor information sharing between healthcare professionals is one of the major causes of preventable death or disability during hospital admissions, and a leading cause of adverse surgical events. $^{56}$ Although responsibility for care 
is shared between medical teams, there is a significant asymmetry in neurosurgical expertise ${ }^{7}$ and it is therefore our duty to ensure that the advice we provide is correctly recorded and appropriately implemented.

Traditional verbal and paper-based handover and referral systems have inherent flaws, which can impact on patient care and lead to heightened medicolegal risk for the referring and receiving teams. Verbal handovers have been shown to result in poor documentation, and the significant loss and misinterpretation of information. ${ }^{7-9}$ Similarly, paper-based systems suffer from illegibility, inadequate paper-trails, incomplete, missing or delayed information, and loss of patients within the system. ${ }^{10}$ This is compounded by staff changes from one shift to the next, which can lead to amplification of errors and also unavoidably results in referrals being revisited by clinicians who have not seen the original patient personally, and to whom the patient has been handed over. ${ }^{11}$

Online referral systems (ORSs) have been shown to be superior to traditional phone and paper-based referrals in neurosurgery: documentation is greater, since all important information is mandatory; the rate of duplication of information during handover is reduced ${ }^{10}$; the response rate to referrals is higher; communications are time-stamped from a designated individual, and phone calls to the on-call neurosurgery registrar are reduced which improves continuity of patient encounters and time management for all parties. ${ }^{10} 11$ Moreover, thorough documentation of patient notes via ORS offers both referring and receiving institutions medicolegal security during patient transfer. ${ }^{10}$ ORS also provides a valuable data capture tool for audit and quality improvement.

There are several ORS databases currently in use in the $\mathrm{UK}^{10-12}$ with a steady increase in the number of neurosurgical units changing their traditional telephone referral process to an online one as the primary way of referring a patient for a neurosurgical opinion. This suggests a change in attitude towards online referrals and that the logistical and infrastructural difficulties initially encountered have been overcome. ${ }^{10}$ There is however very little in the literature about the experience of those referring to neurosurgery and how these changes have affected their practice and perceptions.

The aim of this study is to ascertain the opinions that referring healthcare professionals have of our online referral process and how they feel it impacts patient management. We also want to gather their perceptions of the interaction with the on-call neurosurgical registrars and investigate how our referral process can be improved.

\section{METHODS}

Our ORS was built in 2008 and was designed to receive and manage urgent patient referrals and transfers to the neurosurgical unit at a major teaching hospital in the UK. ${ }^{10}$ This system is not used for elective referrals. It was initially designed to allow data capture and retrieval of patient information within our department. The data were entered by the on-call neurosurgical registrar while discussing the referral over the phone. This was time consuming for both the referrer and the neurosurgical registrar. In 2015, the system was therefore upgraded to allow referrers to have direct access locally following a self-registration process using their professional membership number. More importantly, referrers became able to submit a new referral online without needing to go through the on-call neurosurgery registrar. They were also able to access the outcome of their referral and enter updates in the journal section of the system and upload files such as pdf documents and photographs. Submitted referrals are now instantly received by our department. Updates are visible to the referring hospital in real time with the date and time of the updates being automatically recorded. All relevant healthcare professionals within the neurosurgical department are able to access, review and respond to referrals on the system. The system also highlights pending and updated referrals. Imaging is linked and viewed separately via the picture archiving and communications system. All our referring hospitals use this ORS as the primary way of referring patients to our department, compared with only three hospitals when the system was first launched in $2008 .{ }^{10}$ Our department is increasingly reliant on this ORS to provide neurosurgical advice, with the objective of limiting phone calls to true life-threatening emergencies only.

The first part of our study was performed in October 2014 and again in February 2016 when we analysed the number of phone calls made to the on-call neurosurgical phone in order to assess the impact that the above mentioned changes have had on the number of calls we receive. For the second part of our project, we used an online app (https://www.quicktapsurvey. com) to conduct a survey in 15 hospitals that regularly refer patients to our department over a period of 5 days in May 2016. The survey consisted of 15 questions (table 1) and was designed by a senior neurosurgical registrar (MA) and the senior consultant who was also involved in the creation of the ORS (CT). Sixteen medical students were in charge of disseminating the survey during regular working hours and out of hours. They approached doctors of all grades (foundation year doctors to consultant grades), nurses and physiotherapists from a wide range of medical and surgical specialties. The respondents were selected randomly and those who confirmed being familiar with ORS were asked to complete our survey using the app on the student's smartphone. The medical student conducting the survey was therefore able to answer any questions the respondents might have instantly.

\section{RESULTS}

We observed a dramatic $88 \%$ decrease in the number of phone calls made to our department's on-call registrar, 

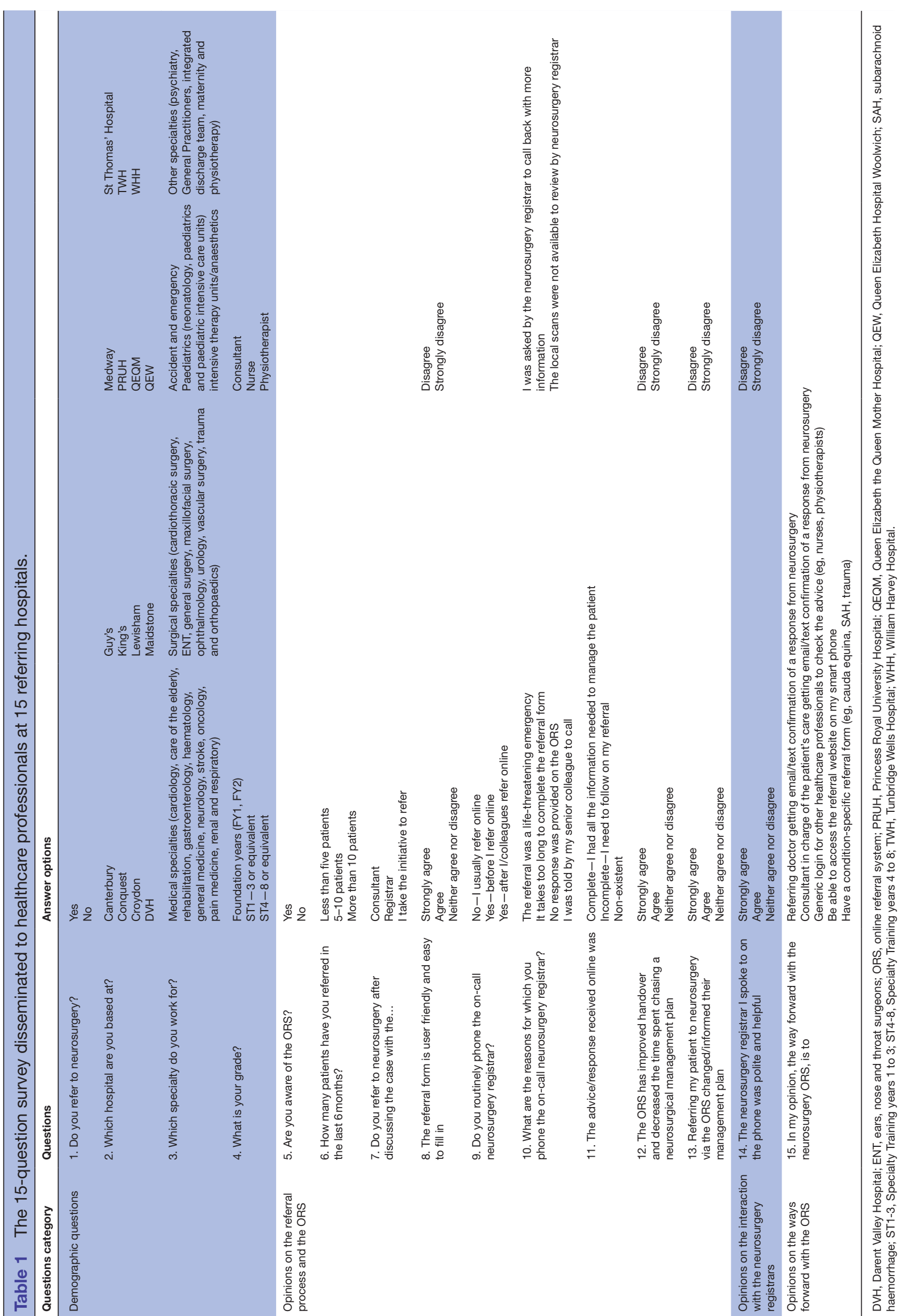


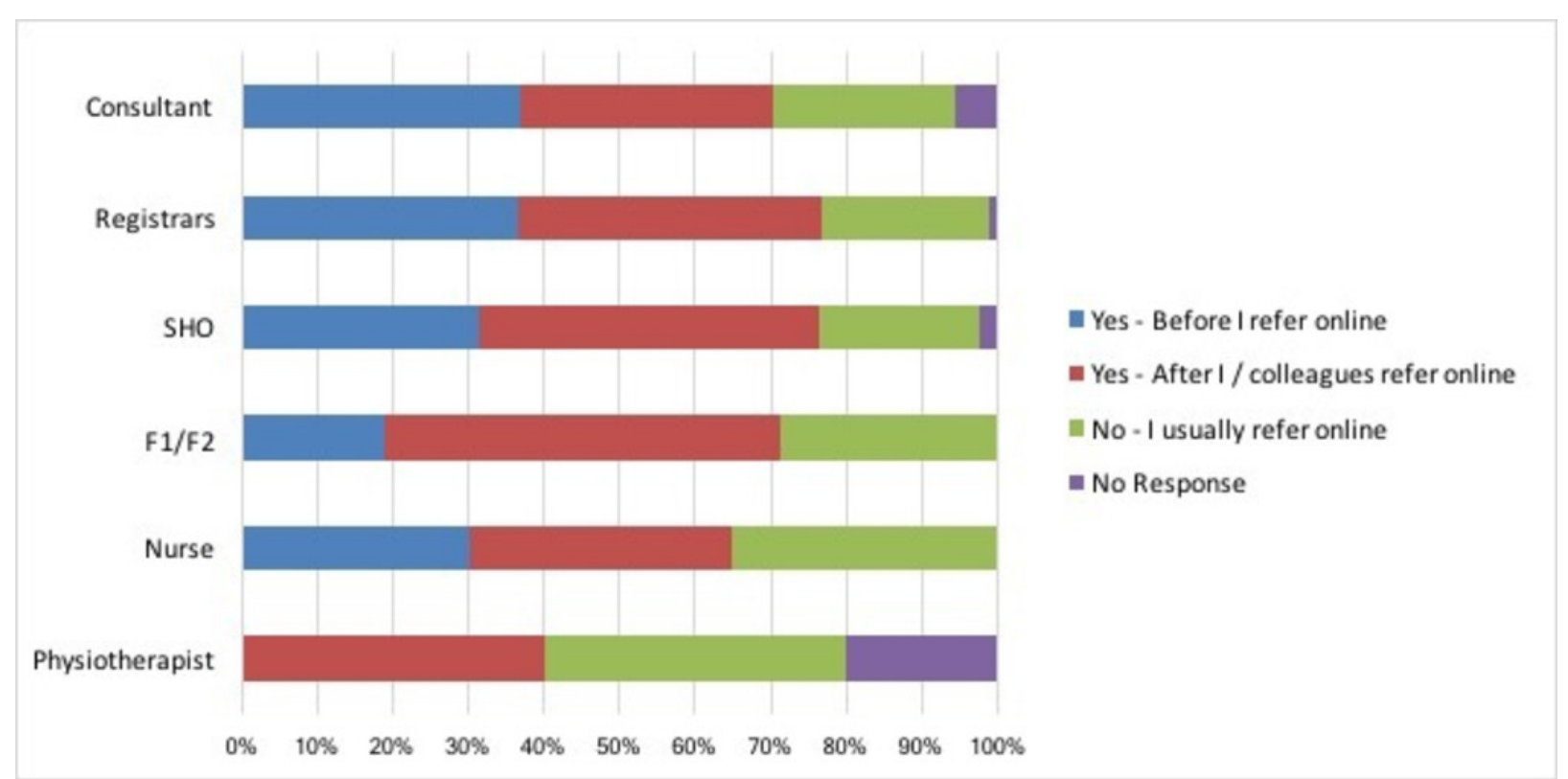

Figure 1 Grades of the $74 \%$ of referrers who continue to phone the on-call registrar before or after making an online referral. F1/F2, Foundation Year 1/2 ; SHO, Senior House Officer.

from 11683 /month in October 2014 to 2581 /month in February 2016 This reduced the time referrers were put on hold before their call was taken. Similarly, the total number of unanswered calls decreased from almost $70 \%$ in October 2014 to just under 9\% in February 2016.

Our survey gathered the opinion of 641 healthcare professionals from 15 referring hospitals. The average number of respondents per hospital was 43 with a range of 18-104 respondents. There was a good distribution across a large number of medical specialties $(\mathrm{n}=290,45 \%)$, accident and emergency $(\mathrm{n}=129,20 \%)$, surgical specialties $(n=117,18 \%)$, paediatrics $(n=50$,
$8 \%)$, intensive therapy unit/anaesthetics $(n=46,7 \%)$ and other specialties $(n=9,1 \%)$. All medical grades were represented: foundation year trainees $(n=162$, $25 \%)$, ST $1-3$ or equivalent $(n=182,28 \%)$, ST $4-8$ or equivalent $(\mathrm{n}=97,15 \%)$, consultant $(\mathrm{n}=68,11 \%)$ as well as nurses $(\mathrm{n}=102,16 \%)$ and physiotherapists $(\mathrm{n}=30,5 \%)$.

Questions 1-4 were mandatory and the subsequent questions were answered by $95 \%$ of respondents on average (range of 91\%-99\%). Of the 641 total respondents, $519(81 \%)$ stated they refer patients to neurosurgery. The results of questions 5-15 will therefore be

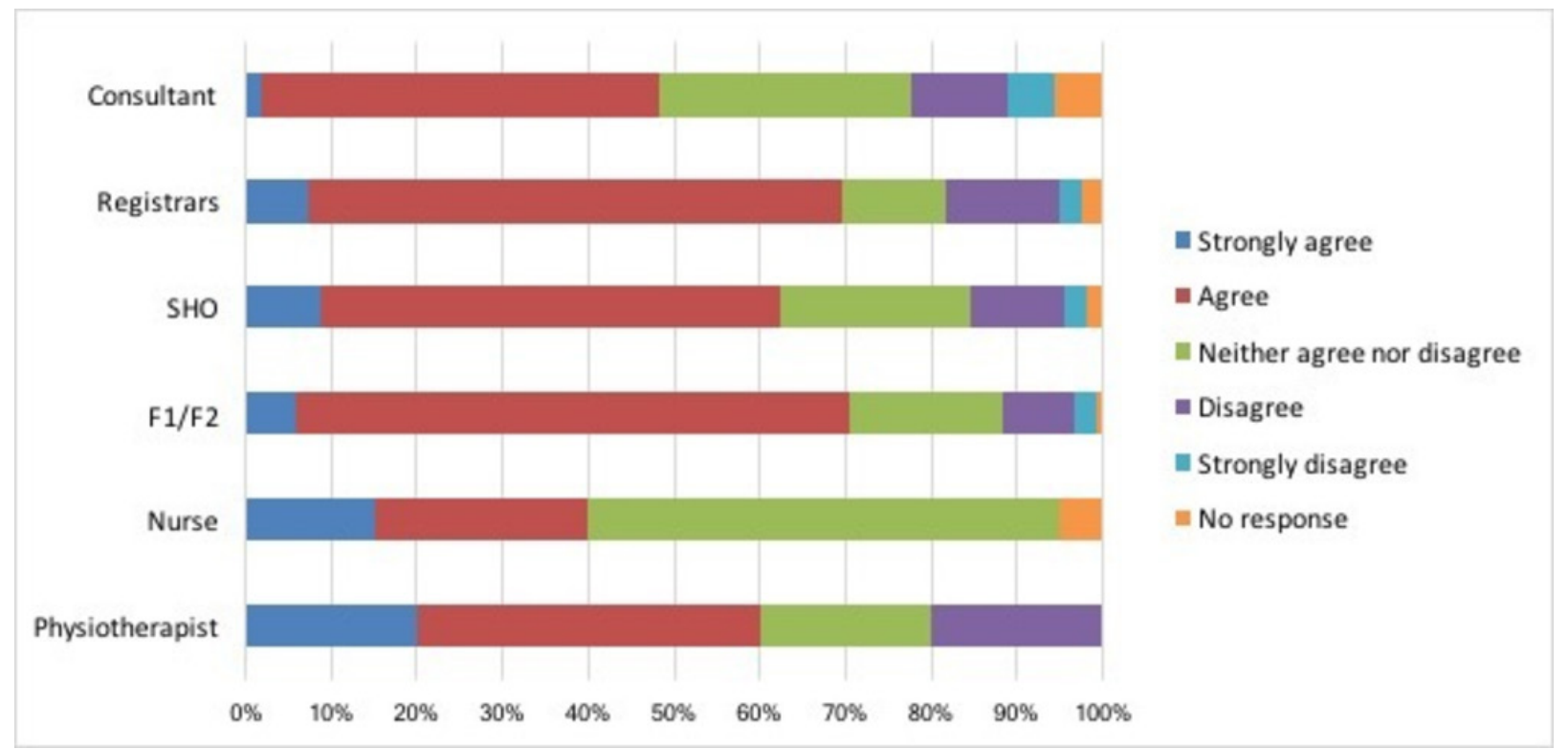

Figure 2 Opinion of referrers by grade on the effect of the online referral system on patient care based on the answers provided to the statement: "Referring my patient to neurosurgery via the online referral system changed/informed their management plan". F1/F2, Foundation Year 1/2 ; SHO, Senior House Officer. 


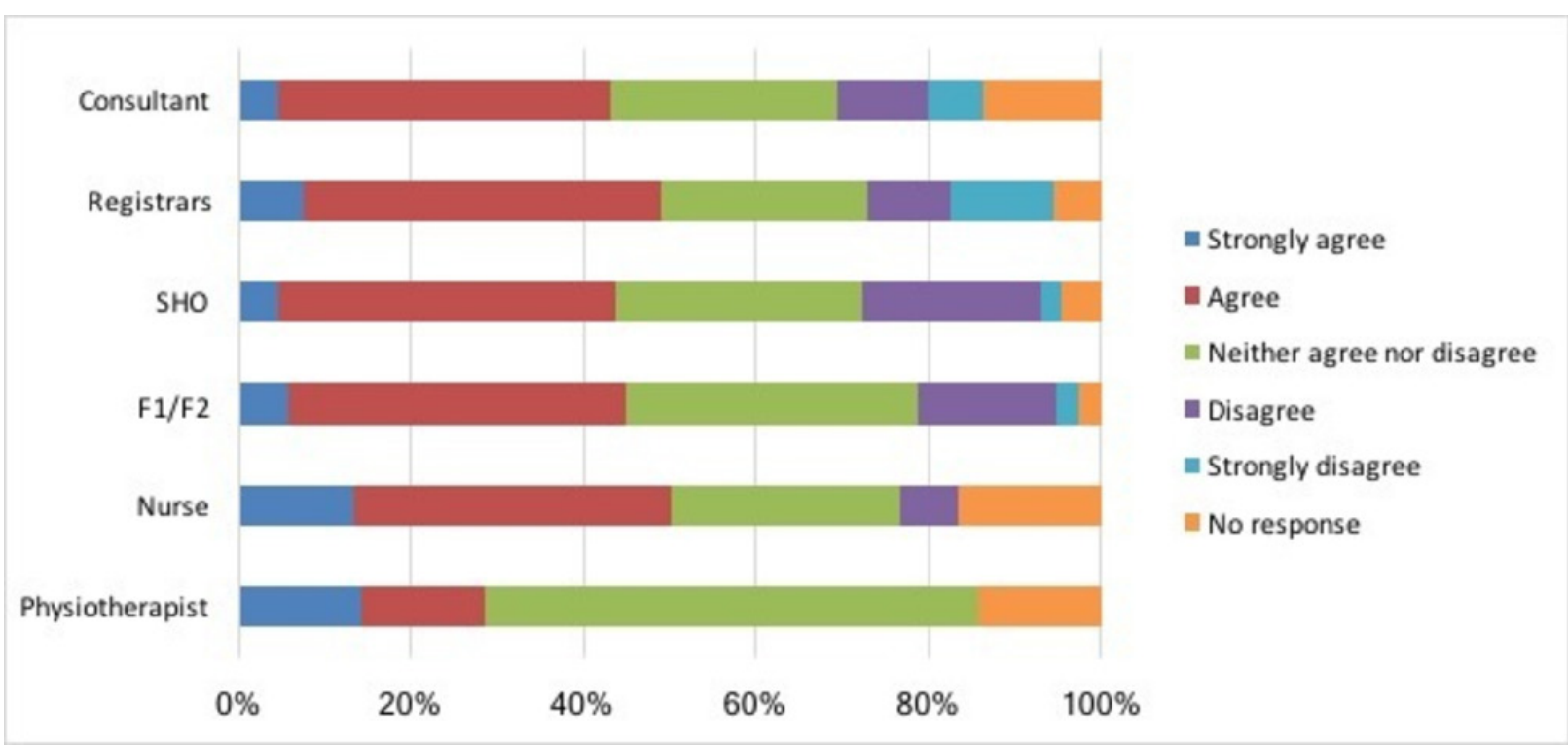

Figure 3 Opinion of referrers by grade on their interaction with our on-call neurosurgery registrars based on the answers provided to the statement "The neurosurgery registrar I spoke to on the phone was polite and helpful". F1/F2, Foundation Year 1/2; SHO, Senior House Officer.

based on those who refer to neurosurgery, excluding any non-respondents to a given question.

\section{Opinions of the referrers on the referral process and the ORS}

A large number of respondents were aware of our ORS $(\mathrm{n}=476,92 \%)$. The majority $(\mathrm{n}=321,62 \%)$ stated referring less than five patients to our neurosurgical department in the 6 months preceding the survey, followed by $5-10$ patients $(\mathrm{n}=144,28 \%)$ and more than 10 patients referred $(n=51,10 \%)$. While registrars $(46 \%)$ and consultants $(82 \%)$ would take the initiative to refer patients for a neurosurgical opinion, the majority of foundation trainees $(85 \%)$, ST1-3 grades $(73 \%)$, nurses $(72 \%)$ and physiotherapists $(86 \%)$ would first discuss with a senior colleague, be it a registrar or a consultant.

On average, $54 \%$ of referrers agreed or strongly agreed that our ORS is user friendly and easy to fill in especially foundation trainees $(58 \%)$ and ST1-3 grades $(57 \%)$. Despite this, $74 \%$ of the respondents would still phone the on-call neurosurgery registrar either before making an online referral $(32 \%)$ or after a referral has been submitted ( $43 \%$ ) while $26 \%$ stated they would only refer online (figure 1). Three main reasons were identified: $44 \%$ of respondents believed their call to be related to a life-threatening emergency, $26 \%$ stated they called because no response was provided on the ORS and $19 \%$ were merely executing seniors' requests.

Just below half the referrers reported that the responses provided in our ORS were complete $(47 \%)$ whereas $45 \%$ felt the answers were incomplete or non-existent $(8 \%)$. When those results were assessed based on the grade of the referrers, three groups had outstanding results compared with the cohort overall. The majority of referring consultants and physiotherapists felt that the responses provided on the ORS were incomplete $(41 \%$ and $86 \%$, respectively) whereas $70 \%$ of the nurses who referred patients to neurosurgery reported a complete response to their request.

To the statement 'the ORS has improved handover and decreased the time spent chasing a neurosurgical management plan', the responses were almost evenly spread into the three given options with a small majority either agreeing or strongly agreeing with this assumption (37\%), while $33 \%$ had a neutral opinion and $31 \%$ disagreed or strongly disagreed with the statement. The same three groups of consultants, nurses and physiotherapists had outstanding results, providing a neutral response at $33 \%$, $43 \%$ and $67 \%$,respectively. Documentation on the ORS was considered helpful in informing patients' management plan by $62 \%$ of referrers on average, with a breakdown as follows: foundation year trainees $(70 \%)$, ST1-3 $(62 \%)$, ST4-8 (66\%), consultants (46\%), physiotherapists (42\%) and nurses (33\%) (figure 2).

\section{Opinions of the referrers on the interaction with the neurosurgery registrars}

Just under half the respondents $(48 \%)$ either agreed or strongly agreed that the neurosurgical registrars they spoke to over the phone were polite and helpful, while $21 \%$ disagreed or strongly disagreed and $31 \%$ neither agreed nor disagreed with this statement. When the results were grouped according to the referrers' grades, half of the number of consultants and registrars surveyed were satisfied with their interaction with the on-call neurosurgery registrars $(50 \%$ and $52 \%$, respectively). However, more junior colleagues seemed less pleased with their interaction with the neurosurgery registrars, with $54 \%$ of ST1-3\% and $53 \%$ of foundation year trainees either disagreeing or giving a neutral response to this question. Physiotherapists had a more neutral opinion in general (33\%), although without any negative responses provided. In contrast, $60 \%$ 
of the surveyed nurses were satisfied with their interaction with the on-call registrars (figure 3).

\section{Opinions of the referrers on the ways forward with the referral process and the ORS}

Five options to improve our ORS were provided in our survey, with the possibility to only choose one. Half the respondents favoured receiving an email or text message confirming that a response to their referral has been entered on the system $(n=244,50 \%)$. The use of a generic login, allowing other healthcare professionals to check the advice provided by neurosurgery, received the vote of 85 respondents $(17 \%)$. The proposition of sending an email or text message to the consultant in charge of the patient's care, notifying them of the neurosurgical response to the referral, was the third favourite option $(\mathrm{n}=78,16 \%)$. We also offered to supply condition-specific referral forms, such as for cauda equina syndrome or subarachnoid haemorrhage, to guide the referrer in providing the information required to provide an informed opinion, but this only appealed to $9 \%$ of the respondents $(n=46)$. The least favourite option was the possibility to access the ORS on a personal smartphone $(\mathrm{n}=36,7 \%)$.

\section{DISCUSSION}

The impact of using ORS on neurosurgical registrar burden has been explored by several authors ${ }^{10-13}$ but little is known about the perceptions and opinions of the primary users of these systems: the local referrers. We have conducted the first regional survey in the UK in order to gain a better understanding of the way referring colleagues perceive our referral process and the way healthcare professionals and patients benefit from using our ORS. We also set out to probe the opinion referring colleagues have of their interaction with our registrars and discuss their opinions on the way to improve our existing ORS to allow it to better suit their needs and render it more efficient.

While our colleagues seem aware of the referral process we currently have in place, the majority $(74 \%)$ would still regularly phone the on-call neurosurgery registrar either before or after making an online referral. Our data offer two potential explanations: (1) referrers tend to perceive the neurosurgical conditions they encounter as being life-threatening emergencies and/or (2) we are not perceived as being efficient enough in providing responses to the referrals made online. The willingness of referrers to discuss a case and its subtleties with another human being over the phone instead of going through an online process is also a possible explanation for the high number of phone calls we continue to receive. This is however beyond the scope of our study but it would benefit from further research.

Why are neurosurgical conditions often perceived as being life-threatening? Unlike most specialties, there are no national guidelines for undergraduate neurosurgery teaching in the UK. ${ }^{14}$ Medical students and junior doctors are therefore unlikely to interact with our specialty until they face patients with neurosurgical conditions while on a medical, surgical or accident and emergency (A\&E) job. Indeed, a recent study, of over 350 students at nine UK medical schools, showed that one-third of final year students have difficulty in knowing when a neurosurgery referral is required. ${ }^{15}$ Our data have also highlighted the fact that individual healthcare professionals tend to refer a very small number of patients to neurosurgery (less than five patients over a period of 6 months). A referrer is therefore unlikely to encounter a neurosurgical condition more than once, thus potentially reducing their ability to independently manage similar patients in the future as well as limiting their learning process. We therefore feel that we need to adopt a more educationally tailored approach to neurosurgical referrals. By this, we mean that the potentially limited neurosurgical experience of our referring colleagues needs to be taken into account when discussing a referral. We argue that guiding colleagues into making accurate referrals by taking the time to provide additional explanation if necessary or by increasing their awareness of available guidelines and policies, such as the Society of British Neurological Surgeons 'treat and transfer' policies, ${ }^{16}{ }^{17}$ for example, is likely to serve a triple purpose: improve the quality of the referrals we receive, promote a better understanding of neurosurgical conditions and ultimately, improve patient care. We do however acknowledge that this can be difficult to achieve in an overstretched neurosurgical service and that it is likely to require a significant cultural change.

Our survey was not designed to audit our response process to the referrals we receive online but we have nevertheless reflected on the potential factors contributing to our referring colleagues' dissatisfaction. Our current ORS highlights both newly submitted and updated referrals in bold. However, the former appear at the top of the list whereas the latter do not. Older referrals with ongoing questions from our referring colleagues are often only reviewed and answered after a follow-up call is received. While the first part of our audit shows that we have managed to significantly decrease the number of phone calls we receive since the modifications implemented in 2015, the on-call phone number was still dialled 89 times a day on average in February 2016. This is likely to divert the attention of the on-call registrar from answering new referrals towards dealing with older ones, resulting in further follow-up calls. The results of the present survey have made us realise the need for better ways of highlighting unresolved referrals and we are in the process of updating our ORS in that respect. An encouraging two-thirds of respondents did however state that the use of our ORS was helpful in informing patients' management plan hence reinforcing our belief in the importance of optimising our ORS to better suit the needs of our referrers and match their expectations.

It is also worth considering the work of Storey and Webster $^{9}$ when reflecting on the above. They identified inconsistent advice and management of patients who are not accepted for transfer as the two key sources of referrer 
dissatisfaction. The former relies heavily on the experience of the registrar providing the advice and on the opinion of the on-call consultant. A given plan might be altered after it is discussed with a more senior colleague or if on-call duties are handed over to a new consultant. Although not specifically addressed by our data, the use of a real-time ORS is likely to contribute to decrease the incidence of inconsistent advice by ensuring written documentation of all the conversations. This allows both referring and neurosurgical teams to have instantaneous access to the conversation trail and avoids misinterpretation of the advice, a problem often reported with paper-based and telephone-based referrals. It is however important that we strive to provide such responses in a timely manner as discussed above. Moreover, we also acknowledge that the use of an ORS is not a stand-alone solution to referrers dissatisfaction and that the human factors discussed throughout this paper are also of paramount importance. Patients not accepted for transfer to a neurosurgical unit present the referring team with ongoing clinical care for conditions they might not be familiar with. Local teams are also left to update patients and families without, sometimes, being aware of the rationale behind a specific management plan. They may also not have insight into why some seemingly similar cases are accepted for transfer to a neurosurgical unit and others are not. Anecdotal evidence from the authors suggests that a brief explanation for the rationale in cases where the patient is not accepted for transfer goes a long way towards fostering confidence in the referring team.

This brings us to discussing the second aim of our survey: the opinion our referring colleagues have of their interaction with the on-call neurosurgery registrars. The survey returned mixed opinions with almost half the respondents perceiving our registrars as being polite and helpful whereas the other half did not. Bradley et al report that neurosurgery is among the subset of specialties that are perceived negatively by referrers alongside radiology, general surgery and cardiology. ${ }^{18}$ Our survey only included one question on this subject and, in retrospect, would certainly have benefited from a more elaborated questionnaire to identify factors that are specific to our practice. Rude and dismissive behaviour in the workplace should not be tolerated nor excused so, with that in mind, we sought advice from the literature to understand potential causes and identify ways to reduce and ultimately banish this behaviour. Factors such as lack of communication skills, physical and mental fatigue and poor understanding of the challenges encountered by other specialties were the recurrent themes we came across.

Some authors have sought to find solutions to unprofessional behaviour by promoting education and training for medical students and newly qualified doctors. ${ }^{19-21}$ This training has been reported to increase the students' self-confidence, but there remains little evidence that these skills can be transferred to clinical settings. ${ }^{19}$ Since 2010, junior neurosurgical residents in the United States start their training by attending boot camps to enhance their psychomotor skills and also their professionalism and communication skills. ${ }^{22}$ The published results suggest that the courses are effective and well accepted among the residents. ${ }^{22}$ The boot camp approach has more recently been adopted in the UK. ${ }^{23}$

Fatigue is well known to negatively impact the safety and performance of healthcare professionals. ${ }^{24-26}$ What is perhaps less known is that it can also significantly impact their affective state. ${ }^{27}{ }^{28}$ Saadat et al evaluated the impact of partial sleep deprivation on the mood and the cognitive skills of 21 paediatric anaesthesiologists at a tertiary care children's hospital during a regular non-call day and following a 17 hours' overnight shift. ${ }^{27}$ They demonstrated that the lack of sleep significantly affected tension, anger, fatigue, confusion, irritability and feeling jittery $(\mathrm{P}<0.05)$. Vigour, energy and confidence were also significantly decreased and the paediatric anaesthesiologists were found to be less 'talkative' after a night shift $(\mathrm{P}<0.05)$. It is not difficult to see how these data can be extrapolated to the currently over stretched UK healthcare system.

To underline the importance of relationships between neurosurgery and external departments, it is worth noting the results of a Canadian qualitative study on the perception of neurosurgeons by patients. While competence and qualifications formed part of a positive perception, a significant component of patient confidence came from positive referral from another clinician. ${ }^{29}$ The opinion colleagues have of one another does influence patient perception and also impacts on the way medical professionals interact with each other. Individuals need to appreciate the 'on the ground' challenges faced by one another in order to be able to work together efficiently. Pressures faced by A\&E departments are well covered by the media, but less is known about pressures in acute medical specialties, for example. ${ }^{30}$ In order to provide efficient and timely tertiary management, a tight cooperation is required between district general and teaching hospitals. Encouraging such cooperation can be initiated at an individual level by promoting and encouraging events such as taster days, cross-specialty teaching and social events.

The final objective of our survey was to identify ways to improve our ORS. Our referring colleagues have expressed the wish of receiving an email or text confirmation when a response to their referral is entered in the system as the main way of improving our current referral system. We have been working on implementing this following the completion of the survey. In addition to the improvements discussed above, and in view of the lack of exposure to neurosurgery during and after medical school, we are also working on a more tailored referral system offering standardised condition-specific referral forms to help referrers with limited neurosurgical knowledge provide relevant information to allow timely processing of their referrals. Perhaps, the main way forward with ORS would however be to use a universal referral system at a national level with an accompanying 
training module to increase the familiarity of referring healthcare professionals with common and life-threatening neurosurgical conditions.

\section{CONCLUSION}

We have conducted the first large-scale regional survey in the UK to assess opinions on our ORS from the referrers' point of view. Our results confirm that referrers feel that using our ORS positively impacts patient care but that it remains in need of improvement in order to better suit our colleagues' needs when it comes to managing neurosurgical patients.

Although no other studies exist to allow accurate comparison, we believe that our experience is likely to be similar to that of other neurosurgical units across the UK. By openly discussing both the satisfactory and the less positive results elicited by our survey, we hope to promote a change in the way neurosurgical referrals are dealt with. The promotion of education while discussing referrals with colleagues who are likely to have minimal exposure to neurosurgery as well as taking steps to mitigate the effect of adverse workplace human factors would be big steps towards ensuring that we achieve the common goal of neurosurgeons and referrers alike: a high standard in patient care.

Contributors MA and CMT were involved in conception and design of the project and also in the interpretation of data. JJN, SD, NK, NA, DC, FH, TJ, NK, DM, SM, SO, $\mathrm{OP}, \mathrm{IR}, \mathrm{RS}, \mathrm{HT}$ and BV were involved in the acquisition of data. MA, JJN, SD and R-MDS drafted the manuscript. MA, JJN, SD, BC, R-MDS and CMT were involved in revising the manuscript and approved the version published.

Competing interests None declared.

Provenance and peer review Not commissioned; externally peer reviewed.

Data sharing statement Extra data are available by emailing meriem. amarouche@gmail.com.

Open Access This is an Open Access article distributed in accordance with the Creative Commons Attribution Non Commercial (CC BY-NC 4.0) license, which permits others to distribute, remix, adapt, build upon this work non-commercially, and license their derivative works on different terms, provided the original work is properly cited and the use is non-commercial. See: http://creativecommons.org/ licenses/by-nc/4.0/

(C) Article author(s) (or their employer(s) unless otherwise stated in the text of the article) 2017. All rights reserved. No commercial use is permitted unless otherwise expressly granted.

\section{REFERENCES}

1. Lee RS, Woods R, Bullard M, et al. Consultations in the emergency department: a systematic review of the literature. Emerg Med $\mathrm{J}$ 2008;25:4-9.

2. Reid C, Moorthy C, Forshaw K. Referral patterns: an audit into referral practice among doctors in emergency medicine. Emerg Med J 2005;22:355-8.

3. Croft SJ, Barnes J, Ginnis C, et al. An evaluation of the referral process in the emergency department. Emerg Med $\mathrm{J}$ 2014;31:827-32.

4. Woods RA, Lee R, Ospina MB, et al. Consultation outcomes in the emergency department: exploring rates and complexity. CJEM 2008;10:25-31.

5. Wu RC, Tran K, Lo V, et al. Effects of clinical communication interventions in hospitals: a systematic review of information and communication technology adoptions for improved communication between clinicians. Int J Med Inform 2012;81:723-32.

6. Nagpal K, Arora S, Vats A, et al. Failures in communication and information transfer across the surgical care pathway: interview study. BMJ Qual Saf 2012;21:843-9.

7. Cartmill M, White BD. Telephone advice for neurosurgical referrals. Who assumes duty of care? Br J Neurosurg 2001;15:453-5.

8. Bhabra G, Mackeith S, Monteiro P, et al. An experimental comparison of handover methods. Ann R Coll Surg Engl 2007;89:298-300.

9. Storey M, Webster S. Giving ourselves a head start: improving the quality of documentation of referrals to neurosurgery. BMJ Qual Improv Rep 2015;4:u203531.w2516.

10. Choo MC, Thennakon S, Shapey J, et al. A web-based referral system for neurosurgery--a solution to our problems? Br J Neurosurg 2011;25:384-7.

11. Matloob SA, Hyam JA, Thorne L, et al. Improving neurosurgical communication and reducing risk and registrar burden using a novel online database referral platform. Br J Neurosurg 2016;30:191-4.

12. Wilson M, Bulters $D$. Response to: a web-based referral system for neurosurgery; a solution to our problems? Br J Neurosurg 2011;25:787.

13. Choo MC, Tolias CM. Response to: a web-based referral system for neurosurgery--a solution to our problems? Br J Neurosurg 2012;26:298.

14. Whitehouse KJ, Moore AJ. Undergraduate teaching of neurosurgery - what is the current practice in the UK and is there a need for improvement? Br J Neurosurg 2015;29:753-7.

15. Skarparis $\mathrm{Y}$, Findlay CA, Demetriades AK. The teaching of neurosurgery in UK medical schools: a message from British medical students. Acta Neurochir 2016;158:27-34.

16. Society of British Neurological Surgeons. The Society of British Neurological Surgeons care quality statement. 2015 http://www. sbns.org.uk/index.php/download_file/view/975/87/2015 (accessed 02 Mar 2017).

17. NICE. Head injury: assessment and early management. $2014 \mathrm{https} / / /$ www.nice.org.uk/guidance/cg176/chapter/1-Recommendations - transfer-from-hospital-to-a-neuroscience-unit2014 Available from: https://www.nice.org.uk/guidance/cg176/chapter/1Recommendations - transfer-from-hospital-to-a-neuroscienceunit2017.

18. Bradley V, Liddle S, Shaw R, et al. Sticks and stones: investigating rude, dismissive and aggressive communication between doctors. Clin Med 2015;15:541-5.

19. Buckley S, Ambrose L, Anderson E, et al. Tools for structured team communication in pre-registration health professions education: a Best Evidence Medical Education (BEME) review: BEME Guide No. 41. Med Teach 2016;38:966-80.

20. Bradley $\mathrm{V}$, Whitelaw $B C$, Lindfield $\mathrm{D}$, et al. Teaching referral skills to medical students. BMC Res Notes 2015;8:375.

21. Lindfield D, Bradley V, Whitelaw B. Teaching and developing referral skills for new doctors. Med Educ 2015;49:1152-3.

22. Fontes RB, Selden NR, Byrne RW. Fostering and assessing professionalism and communication skills in neurosurgical education. J Surg Educ 2014;71:e83-9.

23. deSouza R-M, Walsh D. A career in neurosurgery BMJ careers 2015. $2017 \mathrm{http}: / /$ careers.bmj.com/careers/advice/view-article.html?id= 20022742 (accessed 3 Mar 2017).

24. Olson EJ, Drage LA, Auger RR, et al. physician performance, and patient safety. Chest 2009;136:1389-96.

25. Lockley SW, Barger LK, Ayas NT, et al. Effects of health care provider work hours and sleep deprivation on safety and performance. Jt Comm J Qual Patient Saf 2007;33(11 Suppl):7-18.

26. Pilcher JJ, Huffcutt Al. Effects of sleep deprivation on performance: a meta-analysis. Sleep 1996;19:318-26.

27. Saadat H, Bissonnette B, Tumin D, et al. Time to talk about workhour impact on anesthesiologists: The effects of sleep deprivation on Profile of Mood States and cognitive tasks. Paediatr Anaesth 2016;26:66-71.

28. Wali SO, Qutah K, Abushanab L, et al. Effect of on-call-related sleep deprivation on physicians' mood and alertness. Ann Thorac Med 2013;8:22-7.

29. Samuel N, Shamji MF, Bernstein M. Neurosurgical patients' perceptions of the "surgeon+": a qualitative study. J Neurosurg 2016;124:849-53.

30. Royal College of Physicians of Edinburgh. Pressures in acute medical specialities. 2017 https://www.rcpe.ac.uk/college/pressures-acutemedical-specialities (accessed 19 Mar 2017). 